\title{
Evolutionary paths of language
}

\author{
J. Burridge ${ }^{1}$, T. Blaxter ${ }^{2}$ and B. VAux ${ }^{2}$ \\ 1 School of Mathematics and Physics - University of Portsmouth \\ 2 Faculty of Modern and Medieval Languages and Linguistics - University of Cambridge
}

PACS 87.23.Ge - Dynamics of social systems

PACS 89.75.-k-Complex Systems

PACS 89.75.Hc - Networks and genealogical trees

\begin{abstract}
We introduce a stochastic model of language change in a population of speakers who are divided into social or geographical groups. We assume that sequences of language changes are driven by the inference of grammatical rules from memorised linguistic patterns. These paths of inference are controlled by an inferability matrix which can be structured to model a wide range of linguistic change processes. The extent to which speakers are able to determine the dominant linguistic patterns in their speech community is captured by a temperature-like parameter. This can induce symmetry breaking phase transitions, where communities select one of two or more possible branches in the evolutionary tree of language. We use the model to investigate a grammatical change (the rise of the phrasal possessive) which took place in English and Continental North Germanic languages during the Middle Ages. Competing hypotheses regarding the sequences of precursor changes which allowed this to occur each generate a different structure of inference matrix. We show that the inference matrix of a "Norway Hypothesis" is consistent with Norwegian historical data, and because of the close relationships between these languages, we suggest that this hypothesis might explain similar changes in all of them.
\end{abstract}

Introduction. - The last several decades have seen an explosion in cross-disciplinary applications of statistical physics. This work encompasses a wide range of topics, from the motion of crowds [1] and the flocking of birds [2] to the distribution of wealth [3], the behaviour of social systems [4] and, the focus of this letter, the evolution of language [5-9]. Linguistics (the study of human language) seeks to rigorously describe how the building blocks of language may be combined to convey meanings [10]. Each spoken language has a set of sounds or phones which are typically generated by shaping airflow through the vocal tract in various ways. Phones are derived from the underlying inventory of contrastive elements in a language, called phonemes, by a set of phonological rules. Above the level of individual sounds, languages have morphological rules for building words from morphemes (the smallest units of meaning), and syntactic rules for combining words together to form sentences. The world's languages are defined by a vast array of sounds, generalizations (i.e. rules, constraints, and/or associations) and lexicons, which are all constantly evolving, and it is well known to linguists that the structures of social groups, identities, hierarchies and geography play powerful roles in controlling this evo- lution [11,12]. Recognising language as a phenomenon which emerges from interactions between large numbers of individuals, statistical physicists have constructed deliberately minimalist models to understand properties of its evolution $[4-7,13-16]$. Simplicity is also recognised as a virtue in the field of statistical (machine) learning, where sparsity can increase predictive power [17]. This was also the goal of the locus classicus for phonological theory: Chomsky and Halle (1968) [18]. Our aim is to develop a simple model, which is flexible enough to capture a wide range of realistic linguistic processes, and to be calibrated to linguistic data sets which may be time dependent, geographically distributed and/or labelled by social, gender or other factors.

Model definition. - We assume it is possible to write down a detailed inventory of phones, phonological, morphological, and syntactic rules, and lexical entries (with meanings) which fully define each speaker's own version of their language. We refer to this as the full language state of a speaker. This inventory may be viewed as a high dimensional feature vector [17]. Every speaker will occupy a different location in feature space, but groups of speakers may share many features in common, form- 
ing clusters which represent distinct languages or dialects. The process of language evolution is simply the progressive alteration of the components of individual feature vectors.

We divide time into epochs indexed by $t \in\{0,1, \ldots\}$. We consider a population of speakers, and focus on a subspace of their language which has $p$ different linguistic states or variants (possible feature vectors). At each time $t$, each speaker will be in one of these $p$ states. Language evolution is intrinsically coupled to the social and geographical structure of society, and we may account for this by dividing our population into $n$ groups by some criterion or set of criteria (sex, location, social class $[11,12]$ ). Let $N_{i k}(t)$ be the number of speakers in group $i$ who are in linguistic state $k$ at epoch $t$. We define the relative frequency

$$
f_{i k}(t)=\frac{N_{i k}(t)}{N_{i}}
$$

where $N_{i}$ is the size of the $i$ th group. Language evolution can be driven by children inferring altered versions of the linguistic rules which generated the speech they have heard, and by adults switching their linguistic behaviour over time [19]. We allow for the possibility that speakers preferentially select the most common patterns; an optimal strategy if matching language patterns with interlocutors confers some advantage to a speaker. Such non-linearity in decision making has been observed in human social learning [20] and may be modelled by defining an adjusted frequency [13]

$$
v_{i k}=\frac{f_{i k}^{\beta}}{\sum_{j} f_{i j}^{\beta}}
$$

which emphasizes more popular states when $\beta>1$. When $\beta<1$ higher frequencies are reduced, and lower ones emphasised. We call $\beta$ the conformity number. We write $\mathbf{v}_{i}=\left(v_{i 1}, v_{i 2}, \ldots v_{i p}\right)^{T}$ for the set of adjusted frequencies in group $i$. In principle each group can exhibit different levels of conformity to each of the groups it is exposed to, but for simplicity we set all conformity numbers equal.

We assume that the speakers in each group are all exposed to approximately the same primary linguistic data (PLD), and that this experience is captured by an $n \times n$ stochastic (unit row sums) matrix $K$ where $K_{i j}$ is the fractional observational weight placed on group $j$ by speakers in group $i$. We call $K$ the importance matrix. It encodes all information that we wish to capture about social or geographical groupings and their connectivity. We endow each group with a time decaying memory for the conformity adjusted states of other groups

$$
\mathbf{m}_{i}(t)=e^{-1 / \tau} \mathbf{m}_{i}(t-1)+\left(1-e^{-1 / \tau}\right) \sum_{j} K_{i j} \mathbf{v}_{j}(t)
$$

Memories are related to current behaviour via a $p \times p$ stochastic inference matrix $\mathcal{S}$ : at each epoch we take the distribution of speakers using each variant to be multinomial with probability vector

$$
\mathbf{p}_{i}(t+1)=\mathbf{m}_{i}(t) \mathcal{S} .
$$

Letting $\mathbf{X}(n, \mathbf{p})$ be a generic multinomial random vector with $n$ trials and probability vector $\mathbf{p}$ we have

$$
\mathbf{N}_{i}(t) \stackrel{d}{=} \mathbf{X}\left(n_{i}, \mathbf{p}_{i}(t)\right)
$$

where $\stackrel{d}{=}$ denotes equality in distribution. This completes the specification of our dynamics.

In this letter we focus on linguistic change which is driven by the inference of grammatical rules from current linguistic patterns observable in the PLD. In this case speaker memories for grammar $k$ may be viewed as the PLD or patterns in the PLD generated by those rules, rather than the rules themselves. Different rule sets may generate similar patterns, so speakers may infer one grammar from the surface forms of another [21]. Typically, inference is carried out by younger speakers as they learn their language [22]. The entries of $\mathcal{S}$ have the following interpretation: subconsciously, a speaker selects the pattern she is going to emulate, and then selects (infers) the rules she believes are behind it. We have, for a speaker in group $i$

$$
\begin{aligned}
\mathbb{P}(\text { attempt to emulate surface forms of } k) & =\left[\mathbf{m}_{i}\right]_{k} \\
\mathbb{P}(\text { infer grammar } \mathrm{j} \text { in attempt to emulate } k) & =\mathcal{S}_{k j}
\end{aligned}
$$

where $\left[\mathbf{m}_{i}\right]_{k}$ denotes the $k$ th component of $\mathbf{m}_{i}$. Grammar selection may therefore be viewed as a two step process in our model. The first step - pattern selection - is driven by conformity, and the second - rule selection by inference from linguistic patterns. Expression (4) follows by considering all possible ways in which grammar $k$ could be inferred by this two step process using probabilities (6) and (7). For other forms of linguistic change like lexical change or vowel shifts its interpretation will alter. In these cases $\mathcal{S}$ may be viewed as a linear reweighting of frequency/conformity based selection probabilities which captures bias effects originating from structural principles like maximal dispersion [23], automation of production [24], ratchet effects [25] (in which changes acquire social momentum), phonetically systematic transmission errors (channel bias) and cognitive predispositions (analytic bias) [26].

We are motivated by the desire to model, in a simple and computationally efficient way, the evolutionary routes of language change amongst populations that tend to conform (find consensus) and are socially and spatially labelled. Our computational implementation requires five lines of vectorized Python [27]. The modelling of language change is by now well established and our model has its roots in existing approaches. A matrix with a similar interpretation to our $\mathcal{S}$ appears in work on the evolution of Universal Grammar [16]. Time decaying speaker memories similar to (3) were studied in the utterance selection model [7], which also allowed linear re-weighting of selection probabilities, and our model of consensus may be seen as a modified majority rule [28]. The emergence of consensus in language, including in spatial settings, is also explored in the naming game $[15,29]$. 


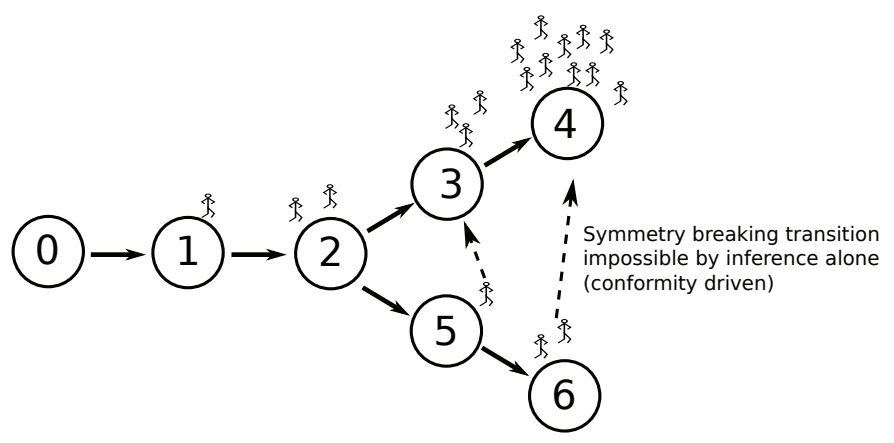

Fig. 1: A linguistic subspace in which stick people are evolving toward one of two grammars. Arrows from state $i$ to $j$ indicate that $\mathcal{S}_{i j}=\epsilon, \mathcal{S}_{j i}=\delta$ with $\epsilon>\delta$ and $\mathcal{S}_{i i}=1-\sum_{j \neq i} \mathcal{S}_{i j}$. Dashed lines indicate transitions not based on inference.

When $\mathcal{S}$ is the identity matrix then the probability that grammar $k$ will be inferred by a speaker in group $i$ is equal to her memory $\left[\mathbf{m}_{i}\right]_{k}$ for the frequency of that grammar. In this case groups of speakers can become fixed in one grammar state when all others have been forgotten. Fixation occurs because it is impossible to recreate any new grammars by mistaken inference. In the case $\beta=1$ this process generates neutral evolution [30,31], where variants in future generations appear with a probability that is proportional to their frequency in previous generations.

Under some circumstances it is useful to consider the large population (deterministic) limit of the model, which for large $\tau$ may be approximated by the following set of coupled differential equations

$$
\begin{aligned}
\dot{\mathbf{m}}_{i} & =\frac{1}{\tau}\left(\sum_{j} K_{i j} \mathbf{v}_{j}-\mathbf{m}_{i}\right) \\
{\left[\mathbf{v}_{i}\right]_{k} } & =\frac{\left[\mathbf{m}_{i} \mathcal{S}\right]_{k}^{\beta}}{\sum_{j}\left[\mathbf{m}_{i} \mathcal{S}\right]_{j}^{\beta}} .
\end{aligned}
$$

From this we see that in the deterministic setting, memory length $\tau$ linearly scales the rate of change of linguistic memory. In the stochastic case it also functions as the window length of a time average, and so dampens fluctuations.

Branching and symmetry breaking. - We now explore a simple inferability matrix which illustrates how a speech community can select from two or more alternative routes of linguistic evolution. Consider the linguistic subspace illustrated in fig. 1, where arrows between states indicate that one grammar is inferable from the other (the direction $i \rightarrow j$ indicates that $j$ is easier to infer from $i$ than vice versa). In fig. 1 most speakers will eventually end up in state 4 or 6 , so in a large and socially disjointed or geographically dispersed speech community we would eventually expect to see both kinds of grammar. However, in a more strongly connected community with sufficiently large conformity number, $\beta$, we may see a symmetry breaking phase transition. To understand this we recall that while

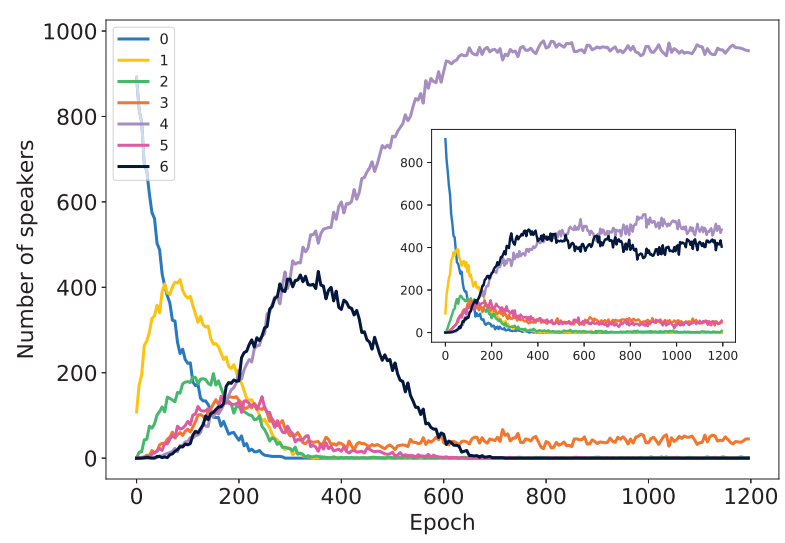

Fig. 2: Symmetry breaking at a grammar branch. Parameter values $\tau=5, \epsilon=0.1, \delta=0.01, \beta=1.05$. Inset plot has $\beta=1.0$.

speakers cannot independently adopt grammars which are not inferable from their native (parental) grammar, they can do so if sufficiently large numbers of the speech community are using the target grammar. In fig. 1, a transition of this kind is illustrated between 4 and 6 . Fig. 2 shows the evolution of a population beginning with grammar 0 . When conformity is sufficiently strong the population arbitrarily select one final dominant grammar. Fig. 2 (inset) shows the evolution of a similar community with lower conformity. Here the symmetry between grammars 4 and 6 is unbroken. At much longer time scales, fluctuations may lead to one state dominating: a different form of symmetry breaking.

We can understand the interaction between conformity and inference transitions by considering a system with two grammar states $X$ and $Y$. Such a system will have an inferability matrix with general form

$$
\mathcal{S}={ }_{Y}^{X}\left[\begin{array}{cc}
X & Y \\
1-\epsilon_{1} & \epsilon_{1} \\
\epsilon_{2} & 1-\epsilon_{2}
\end{array}\right] .
$$

Suppose that our speech community consists of a single group, and let $\mathbf{m}=(v, 1-v)$ then

$$
\mathbf{p}=\left(v\left(1-\epsilon_{1}-\epsilon_{2}\right)+\epsilon_{2}, 1-\epsilon_{2}-v\left(1-\epsilon_{1}-\epsilon_{2}\right)\right) .
$$

In statistical equilibrium the expected values of the entries of this vector are equal to the expectations of the current frequencies of the two grammars in the population. Fluctuations of the true frequencies about these expectations will be of order $N^{-1 / 2}$ where $N$ is the size of the community. For large communities we can therefore approximate the true equilibrium frequencies with their expectations. Replacing the memory values $v$ with their expression in terms of the frequency, $f$, of $X$, we have the equilibrium condition

$$
\frac{f^{\beta}}{f^{\beta}+(1-f)^{\beta}}=\frac{f-\epsilon_{2}}{1-\epsilon_{1}-\epsilon_{2}} \text {. }
$$




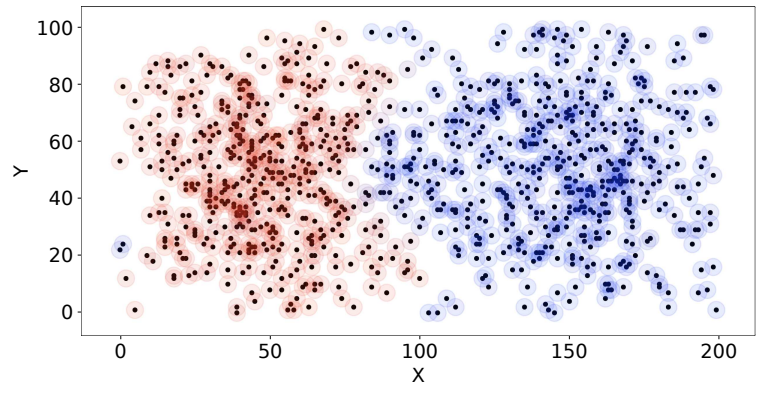

Fig. 3: Equilibrium of two state model (10) where $\epsilon_{1}=$ $0.05, \epsilon_{2}=0.06$ and $\beta=1.3$. Each dot is a group and $K_{i j} \propto \exp \left(-r_{i j}^{2} / \sigma^{2}\right)$ where $r_{i j}$ is the distance between groups $i$ and $j$, and $\sigma=5$. Blue groups are dominated by state $Y$ and red by $X$. The spatial distribution of groups takes the form of two gaussian blobs centred at $(50,50)$ and $(150,50)$.

For low conformity this equation has only one solution, but for sufficiently large $\beta$ a second appears, and the speech community must select one, but we cannot tell in advance which. Even if there is an inferability bias toward one state, conformity may keep the community closer to the other by emphasising it in speakers' memories. If $\epsilon_{1}=$ $\epsilon_{2}=\epsilon$ then we have a symmetry between the two states, and for low conformity the solution is $f=1 / 2$. When

$$
\beta>\frac{1}{1-2 \epsilon}
$$

then this single solution is replaced with two $f=1 / 2 \pm a$ for some $a \in[0,1 / 2]$, only one of which is selected by the community. Hence the term symmetry breaking [32], which we use to refer generically to the appearance of multiple steady states from which the community selects. In thermodynamic systems symmetry breaking generates macroscopic ordering, and is induced by non-linearity in the response of the microscopic constituents of the system to their local environment. The strength of this nonlinearity is typically controlled by temperature. In our case the non-linearity which induces symmetry breaking lies in the relationship between $\mathbf{v}$ and $\mathbf{f}$, and is controlled by $\beta$, which plays a role analogous to inverse temperature in thermodynamic systems.

If we imagine the branch in fig. 1 being part of a much larger network of grammars, then we see that symmetry breaking will cause communities to select one particular route among many through the network. In spatially distributed cases, we might expect to see different routes taken in different places or amongst certain well defined and separated groups, leading to dialects and eventually distinct languages. A simple illustration of how a spatially distributed system can support two equilibrium states is given in fig. 3, where the importance matrix $K$ is structured to model a gaussian spatial interaction kernel between groups. Here we see that a domain wall or isogloss (in linguistic terminology) has formed between two regions
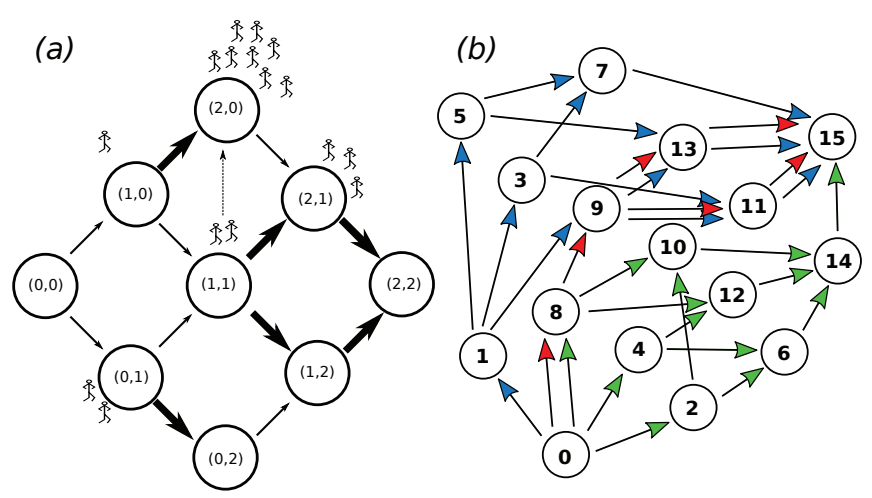

Fig. 4: (a) Transitions in a linguistic subspace of two features; each can exist in $C=2$ contexts. (b) Inference matrices for the three hypotheses. Red: Norwegian, Green: English, Blue: Swedish. An arrow from states $i$ to $j$ implies that $\mathcal{S}_{i j}>\mathcal{S}_{i j}>$ 0. No arrow implies $\mathcal{S}_{i j}=0$.

of dense population. Isogloss dynamics of this kind, which is driven by surface tension, is explored in $[13,14]$.

The timing of linguistic changes. - We now consider the conditions under which two or more linguistic changes which are equally likely to be inferred from the current grammar can spontaneously occur at different times. Often grammatical changes spread though multiple linguistic contexts over time (examples include the spread of do-support through different clause types in Middle English [33] and the spread of the new pronoun chdi between different syntactic contexts in northern Welsh [34]). To capture this we consider features which occur in $C \in \mathbb{N}$ contexts, and simultaneously evolve two equally inferable features $X$ and $Y$. The state of a single speaker is then specified by the integer pair $(i, j)$ indicating that she uses feature $\mathrm{X}$ in $i$ contexts, and $\mathrm{Y}$ in $j$ contexts. Because the spread of a feature from one context to another may be easier to infer than its first appearance, we set the inferability of the first appearance of a features to be $\epsilon_{1}$, with later changes of the form $(i, j) \rightarrow(i+1, j)$ or $(i, j+1)$ having inferability $\epsilon>\epsilon_{1}$. Such acceleration effects may also be caused by social factors [25,35]. A schematic diagram of the square state space in the $C=2$ case is shown in fig. 4 (a). The spread of a feature may be tracked by calculating the average location of the population as its members navigate the square. The appearance of new features may be prevented or delayed if conformity to the current dominant linguistic state overwhelms any inferability bias toward new features. This conformity trapping may be overcome by random fluctuations, where a large enough number of speakers spontaneously adopt the new feature, reducing the dominance of the $(0,0)$ state. A simulation example is shown in fig. 5. Here, both features remain trapped at low levels until fluctuations spontaneously allow one to emerge fully, following the classic S-curve evolution [36,37].

At any moment in time a language has the potential to undergo changes, but their timing appears difficult to 


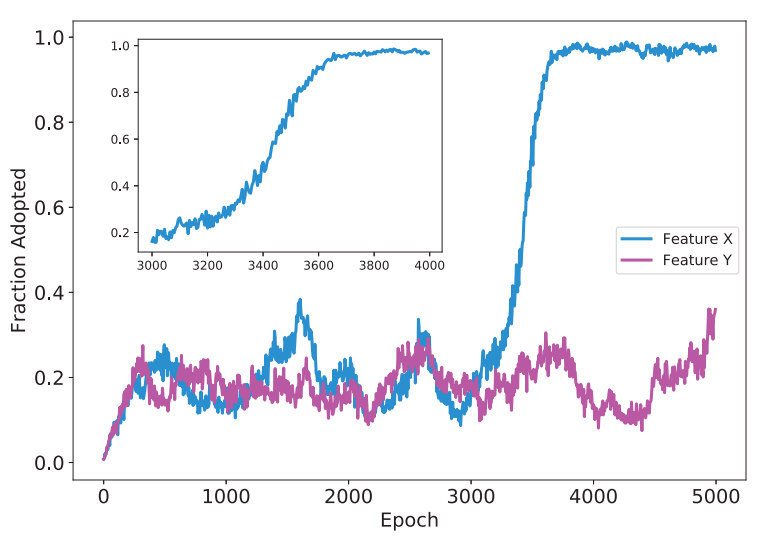

Fig. 5: The evolution of two equivalent features, each of which can occur in $C=5$ contexts. Speakers start in state $(0,0)$. $\epsilon_{1}=0.035, \epsilon=0.075, \delta=0.02, \tau=5$ and $\beta=1.04$ where $\delta$ is the inferability of reverse transitions. Inset shows detail of spontaneous transition in feature $X$.

predict. One proposed explanation for major changes [35] is the trigger event: some relatively small change in part of the linguistic system which facilitates a much wider shift, which may then accelerate. The natural question then is: what triggered the trigger? In our simple model, it is possible for changes to be trapped and waiting to happen with their release an inherently unpredictable event.

The rise of phrasal marking of possessive. - We now use our model to provide a simplified account of historical changes within a linguistic subspace relating to the genitive case. One function of the genitive is to signify possession (e.g. in modern English 'Anna's shoes'). The change we are interested in took place in the grammar of English and Continental North Germanic (Swedish, Danish, Norwegian). In each language, a reanalysis of the genitive ending -(e)s takes place where it goes from having scope over the preceding word to having scope over the preceding phrase; for example

$$
\underbrace{\text { be }[\mathrm{kyng}] \text { is of ffraunce }}_{\text {Middle English }} \rightarrow \underbrace{[\text { the king of France]'s. }}_{\text {Modern English }}
$$

In all four languages, other changes affect the genitive. Among these: all endings are replaced by a uniform ending, -(e)s; all functions of this ending are lost except marking of possessive (loss of 'lexical genitives'); a pattern by which all elements of the phrase are independently marked for genitive ('concord') is lost. These changes are listed and labelled in table 1. Different hypotheses about the relationships among these changes have been proposed, which can be expressed as different inferability matrices. In one hypothesis (the 'English hypothesis' [38]), these latter changes create the ambiguity in surface forms which are necessary for the reanalysis. That is, with reference to table 1, features 1,2 and 3 are needed in order to infer
Table 1: Features for a subspace of Norwegian / Swedish / Danish / English grammar, and numbers of texts in datasets.

\begin{tabular}{c|l|l|l} 
Label & Feature & Abbrev. & Texts \\
\hline \hline 1 & uniform genitive endings & Noun & 3076 \\
2 & loss of concord for genitive & Concord & 933 \\
3 & loss of lexical genitives & Genitive & 1437 \\
4 & -s is a phrasal affix & Affix & NA
\end{tabular}

feature 4

$$
\{1,2,3\} \rightarrow 4 .
$$

In another hypothesis (the 'Swedish hypothesis' [39]) the reverse is true, with the reanalysis happening first in a very restricted context and causing the latter changes

$$
4 \rightarrow\{1,2,3\} .
$$

Our main focus is on a 'Norwegian hypothesis': that just one of these changes, the change to uniform genitive endings, is a necessary precondition for the reanlaysis, and the other changes result from it

$$
1 \rightarrow 4 \rightarrow\{2,3\}
$$

Our model of these processes is simplified in a number of ways. First, we examine only a very small subsystem of the grammar. In reality these changes took place in the context of a much more complex grammatical system and there are other changes, involving other grammatical features, which might be argued to have played a role. Second, our four labelled features are abstractions from more complex sets of sub-features (loss of lexical genitives in different contexts; spread of the uniform -(e)s ending to different classes of words, or even to different individual words; loss of concord in different grammatical contexts and on different classes of words). Third, we assume that inferential changes (from the current grammar) occur in one feature at a time, although speakers can of course jump between any two grammar states provided there are others in those states within the community. These simplifications allow us to describe the model and its output in graspable terms.

We assume that each feature is either present or absent in each speaker, so that each speaker's grammar has a representation of the form $\left(\sigma_{1}, \sigma_{2}, \sigma_{3}, \sigma_{4}\right)$ where $\sigma_{k} \in\{0,1\}$ is the indicator of feature $k$. For brevity we treat this state as the binary representation of an integer $\left(\sigma_{1}, \sigma_{2}, \sigma_{3}, \sigma_{4}\right) \equiv 8 \sigma_{1}+4 \sigma_{2}+2 \sigma_{3}+\sigma_{4}$. Within this state space there are 16 possible grammars and all speakers start in state $(0,0,0,0) \equiv 0$ and eventually reach $(1,1,1,1) \equiv 15$. The set of inferable transitions for each hypothesis is shown in fig. 4 (b).

Fig. 6 shows linguistic data from the Annotated DN online [40], a corpus of medieval Norwegian charters. To generate the data, for each change, a restricted context that 


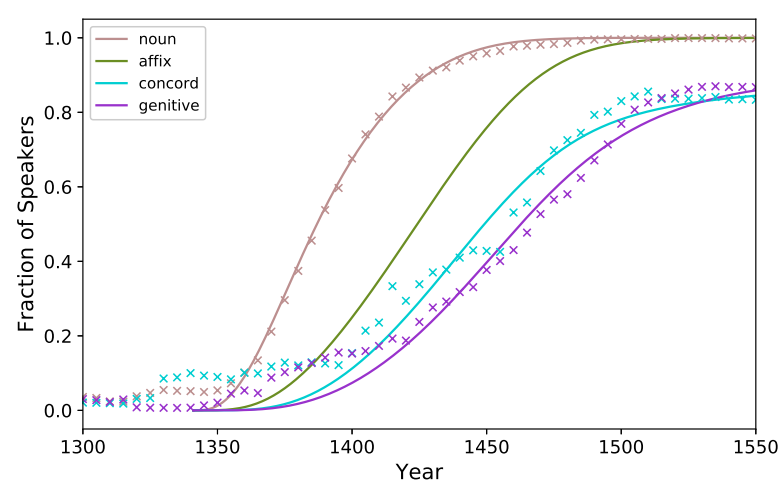

Fig. 6: Solid lines show features evolving according to the Norway hypothesis with $\tau=5$. Parameter values found by least squares optimization $\beta=1.1, \mathcal{S}_{08}=0.19, \mathcal{S}_{89}=0.19, \mathcal{S}_{913}=$ $0.40, \mathcal{S}_{911}=0.25, \mathcal{S}_{1115}=0.06, \mathcal{S}_{1315}=0.11$. The inference values for all reversals (taken all equal) were constrained to match the incomplete transition in concord and genitive, yielding an optimized value 0.03 . Crosses show historical data collected for the same features. Features 2 and 3 have smaller data sets (table 1), and greater ambiguity in identification than 1. Time origin adjusted to match start of historical changes.

could be exhaustively searched and quantified was identified: for uniform genitive endings, patronymics in the form name-GEN+sonr (GEN stands for standard genitive ending and sonr $\equiv$ son); for the loss of concord, agreement between genitive forename and patronymic; and for the loss of lexical genitives, the case taken by the preposition millum 'between'. Feature 4, the change in scope from wordto phrasal-level affix, is very hard to quantify from occurrences in texts as most potential examples exhibit surface ambiguity between the older and newer structures; for this reason, no linguistic data are presented for this feature.

For simplicity we compare our data to the deterministic form (8) of the model. We use calendar years as our unit of time, and set $\tau=5$, equivalent to $\approx 90 \%$ turnover of memory every 10 years. Although $\tau$ cannot be directly measured, we are motivated by the fact that substantial linguistic changes can take place over a decade for speakers across a wide range of ages [19]. To mimic the embedding of the inference matrix (fig. 4 (b)) in a larger network, we incorporated a chain of three precursor states to state 0 , and began the entire community in the first of these. To generate the model fit in fig. 6 we performed a least squares optimization of the conformity number and elements of the inference matrix, restricted to the Norway form. The correspondence between model and data in fig. 6 highlights the plausibility of the Norway hypothesis. However the lack data on the rise of phrasal affix means that a range of inferability values, consistent with the hypothesis, yield similarly good fits to data. We have therefore only established that the structure of $\mathcal{S}$ implied by the hypothesis is consistent with data; there is uncertainty as to the magnitudes of its non-zero entries.
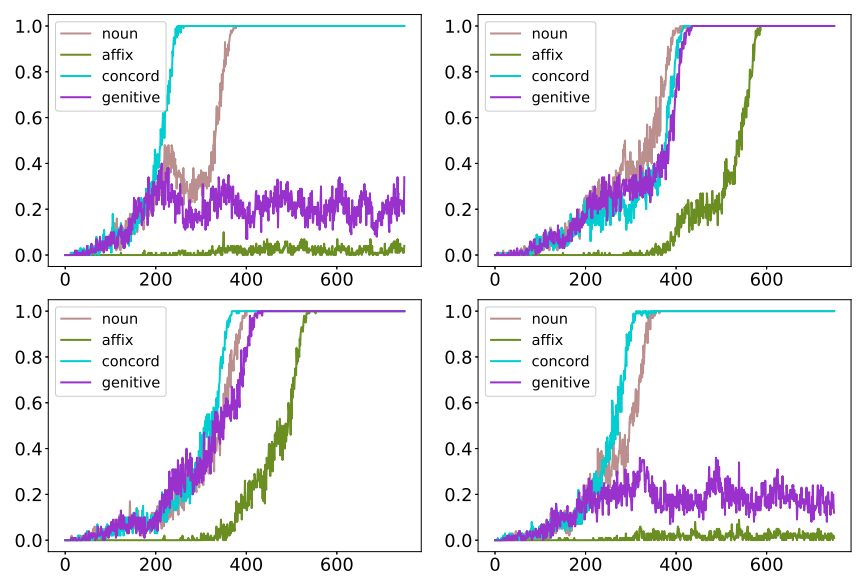

Fig. 7: Evolution of features amongst a single group $(N=$ 100) evolving according to the English hypothesis. In these examples $\tau=20$ epochs, $\beta=1.06$ and all green (English) arrows in fig. 4 correspond to inferability $\epsilon=0.0175$.

We earlier demonstrated that the timing of linguistic changes in the stochastic model can be unpredictable under certain conditions, and we now consider this possibility along with the other two hypotheses. When the inferability values corresponding to each arrow in fig. 4 are small in comparison to the conformity number (in the sense of eq. (13)) then the majority of the population may become trapped by conformity in its current state. In order to escape this state and continue linguistic evolution, they must be freed by random fluctuations. To demonstrate the combined effect of conformity and fluctuations, fig. 7 shows four simulations of the English hypothesis, according to which features 1,2,3 must evolve before the phrasal affix. Although the inference matrix treats each of these preliminary features as equivalent, because of the trapping effects of conformity, the order in which they appear is randomized, as in fig. 5. In the lower conformity, nontrapping regime all three features would evolve at the same time, consistent with the deterministic model. Therefore, in the presence of trapping, if we cannot be sure exactly when the phrasal affix arose in comparison to the other features, then all three hypotheses may be consistent with any observed sequence of changes. We qualify this statement as follows. Trapping of features which are all equally likely to evolve at a given time requires quite a delicate balance between conformity and inferability, and features tend to arise rapidly once they escape the trap. The time intervals between feature appearance can also be highly variable. For these reasons the close fit to experimental data shown in fig. 6 would be hard to achieve in the trapping regime, both in terms of feature timing and S-curve shape. We therefore suggest that the non-trapping case is the more likely of the two explanations that our model provides for observations, at least in the case of Norway.

What are the implications of this for the three hypotheses? Since each hypothesis applies to a different language, 
and Norwegian is the only one for which we currently have high quality quantitative data, then it is possible that all three are correct. However, these languages are closely related: medieval Danish, Swedish and Norwegian were mutually comprehensible and formed a single, continuous dialect area [41]; English was a near geographical neighbour and close relative. Given that such similar changes happened in all these languages at a similar time, it would be surprising if the mechanisms by which they arose were different in each case. We have provided a simple model which matches the historical data for Norway, and supports the Norway hypothesis. While we offer no firm conclusion about the veracity of the three hypotheses, we suggest that the possibility of a single unifying explanation should be considered, requiring further data collection, and a more sophisticated application of our model, involving geographical and cross-linguistic interactions, which may be implemented using the importance matrix, $K$.

Summary. - We have introduced a model of language change which is driven by social conformity and the inference of new linguistic rules from those currently in use. We have explored some essential properties of the model, including symmetry breaking and conformity trapping, and demonstrated how it may be used to quantitatively address a specific linguistic question. The model in principle allows exploration of a variety of different processes and changes in greater detail (possibly requiring a reinterpretation of $\mathcal{S}$ ), including the effects of geographical distribution, and interconnectivity of social groups.

The authors are grateful to the Royal Society for an APEX award (2018-2020), funded by the Leverhulme Trust.

\section{REFERENCES}

[1] Helbing, D., Farkas, I. and Vicsek, T., Nature, 407 (2000) 487.

[2] Bialek W. et. al., PNAS, 109 (2012) 4786.

[3] Yakovenko, V. M. and Rosser J. B. , Rev. Mod. Phys., 81 (2009) 1703.

[4] Castellano, C. Fortunato, S. and Loreto, V., Rev. Mod. Phys., 81 (2009) 591.

[5] Baxter G. J. , Blythe R. A., Croft W. and McKane A. J., Language Variation and Change, 21 (2009) 257.

[6] Schulze C., Stauffer D., and Wichmann S., Communications in Computational Physics, 3 (2009) 271.

[7] Baxter G. J. , Blythe R. A., Croft W. and McKane A. J., Phys. Rev. E, 73 (2006) 046118.

[8] Fort J., and Perez-Losada J, Journal of The Royal Society Interface, 13 (2016) 20160185.

[9] Goncalves B., Loureiro-Porto L., Ramasco J. J. and Sanchez, D., PLoS ONE, 13 (2018) e0197741.

[10] Fasold, R. W. and Connor-Linton J., An Introduction to Language and Linguistics (Cambridge University Press, Cambridge) 2014
[11] Chambers, J. K. and Trudgill, P., Dialectology (Cambridge University Press, Cambridge) 1998

[12] Labov W., Principles of Linguistic Change (Blackwell, Oxford) 1994

[13] Burridge J., Phys. Rev. X, 7 (2017) 031008.

[14] Burridge J., Royal Society Open Science, 5 (2018) 171446.

[15] Baronchelli, A. and Felici, M. and Loreto, V. and Caglioti, E. and Steels, L., Journal of Statistical Mechanics: Theory and Experiment, 2006 (P06014) .

[16] Nowak, M. A. and Komarova, N. L. and Niyogi, P. , Nature, 291 (2001) 114.

[17] Hastie T., Tibshirani R. and Friedman J., The Elements of Statistical Learning (Springer, New York) 1994

[18] Chomsky, N. and Halle, M., The Sound Pattern of English (Harper and Row, New York) 1968

[19] Sankoff, G. and Blondeau, H. , Language, 83 (2007) 560.

[20] Morgan, T. J. H., Rendell, L. E. Ehn, M. Hoppitt, W. and K. N. Laland, Proc. R. Soc. B, 279 (2012) 653.

[21] Hale, M. and Reiss, C., The phonological enterprise (Oxford University Press, Oxford) 2008

[22] YANG, C., Language variation and change, 12 (2000) 231.

[23] Liljencrants, J. and Lindblom, B. , Language, 48 (1972) 839.

[24] ByBeE, J., Language variation and change, 14 (2002) 261.

[25] Lieberson, S., A Matter of Taste:How Names, Fashions and Culture Change (Yale University Press, New Haven) 2000

[26] Moreton, E. , Phonology, 25 (2008) 25.

[27] Van der Walt, S., Colbert, S. C., and Varoquaux, G. , Computing in Science and Engineering, 13 (2011) 22.

[28] Krapivsky, P. L. and Redner, S., Physcial Review Letters, 90 (2003) 238701.

[29] Castello, X. and Baronchelli, A. and Loreto V., The European Physical Journal B, 71 (2009) 557.

[30] Blythe R. A. and McKane A. J., Journal of Statistical Mechanics: Theory and Experiment, 2007 (P07018) .

[31] Kauhanen H. , Journal of Linguistics, 53 (2016) 327.

[32] Blundell S. J. and Blundell K. M., Concepts in Thermal Physics (Oxford University Press, Oxford) 2006

[33] Kroch A. S. , Language Variation and Change, 1 (1989) 199.

[34] Willis D., Journal of Linguistic Geography, 5 (2017) 4166.

[35] Labov, W., Principles of Linguistic Change: Cognitive and Cultural Factors, Volume 3 (Wiley, Chichester) 2010

[36] Blythe R. A. and Croft W. , Language, 88 (2012) 269.

[37] Ghanbarnejad F., Gerlach M., Miotto J. M. and Altmann E. G., J. R. Soc. Interface, 11 (2014) 20141044.

[38] Allen C. L., Genitives in Early English: Typology and Evidence (Oxford University Press, Oxford) 2008

[39] Norde M. , The History of the Genitive in Swedish. A Case Study in Degrammaticalization (Universiteit van Amsterdam, Amsterdam) 1997

[40] Blaxter T., Annotated DN online (http://www.icge. co.uk/dn_online/) 2017

[41] Vikor L. S. , The Nordic languages: An international handbook of the history of the North Germanic languages, edited by BAndLE, O. ET AL., Vol. 1 (de Gruyter, New York) 2002, p. 6-7. 